\title{
Erste Eindrücke vom grazilsten Knochenfräser
}

\section{Eine bestimmte oralchirurgische Indikation perfekt bedienen heißt heute: minimalinvasives Vor- gehen und maximale Schonung der Substanz. Der Oralchirurg Dr. Markus Blume, Brühl, hat den zylindrischen Knochenfräser H255E mit den ausgeprägt grazilen Dimensionen in der Praxis ge- testet. Heißt für ihn grazil auch gut?}

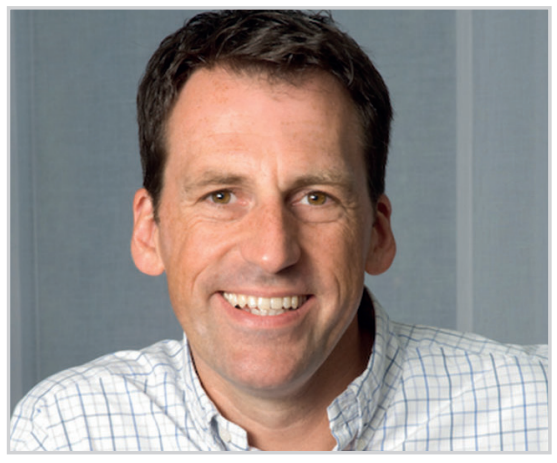

Dr. Markus Blume

Herr Dr. Blume, wie haben Sie bisher Knochengewebe und Zahnhartsubstanz präpariert?

Dr. Markus Blume: Ich benutze seit Jahren kreuzverzahnte Lindemann- und Kugelfräser, wobei mir besonders wichtig ist, scharfe, „frische“ Fräser zu benutzen, die durch die Kreuzverzahnung einen hohen Spanabtrag bieten und den Knochen nicht überhitzen. Für eine schonende Zahnentfernung ist es jedoch oft notwendig, innerhalb einer schmalen Wurzel zu trennen, um das Knochenfach für eine spätere Implantation nicht zu stark zu traumatisieren. Da habe ich mich riesig gefreut, dass Komet nun einen sehr grazilen Fräser, den H255E, anbietet.

Abb. 2

Der zylindrische H255E: hohe Schnittgeschwindigkeit auch im vorderen Arbeitsteilbereich.
Der H255E erschien zur IDS 2015. Ihr erster Eindruck?

Blume: Auf den 1. Blick wird jedem Zahnarzt die kleine Dimension des Instrumentes auffallen. Es ist ja nur 1,2 mm im Durchmesser und $6 \mathrm{~mm}$ lang. Doch obwohl der Fräser so filigran ist, bietet er eine erstaunliche Schneidleistung. Das ist auf die spezielle Kreuzverzahnung mit einem noch größeren Spanraum und längeren Schneidkanten an der Speerspitze zurückzuführen. Die Schneidengeometrie ist durch das zylindrische Arbeitsteil vorgegeben und ermöglicht diese besonders effektive Präparation. Gut zu wissen: Das Instrument funktioniert auf Hand- und Winkelstück gleichermaßen. Ich finde

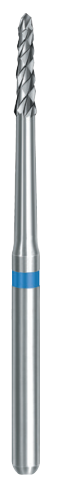

Abb. 3 Der konische H254E: geringere Schnittgeschwindigkeit im vorderen Arbeitsteilbereich (ideal für punktuelles Vorgehen).

außerdem, dass er viel Taktilität zulässt. Bei der Zahnteilung verwende ich am liebsten das rote Winkelstück, hier zeigt sich der Fräser aufgrund der hohen möglichen Drehzahl am effektivsten.

Welchen Unterschied macht denn die Schneidengeometrie konisch oder zylindrisch?

Blume: Viele chirurgisch tätige Praxen kennen vielleicht das ebenfalls grazile, konische Kombinationsinstrument H254E. Der neue H255E ist quasi das zylindrische Pendant des H254E. Beim konischen H254E ist die Schnittgeschwindigkeit im vorderen Arbeitsteilbereich gerin- ger als beim zylindrischen H255E. Hier zeigt die Spitze etwas weniger Schneideigenschaft. Das kann für ein punktuelles Vorgehen von Vorteil sein. Der zylindrische $\mathrm{H} 255 \mathrm{E}$ hingegen ist über die gesamte Länge gleich stark bzw. grazil. Hier wird Knochen- und Zahnhartsubstanz gleichmäßig abgetragen, seine Schnittgeschwindigkeit ist auch im vorderen Arbeitsteilbereich sehr hoch verglichen mit dem konischen H254E. Grundsätzlich ist es aber reine Geschmackssache des Behandlers, welche Eigenschaften er sich von einem Knochenfräser wünscht.

\section{Für was ist der neue Fräser Ihrer Meinung nach indiziert?}

Blume: Ich sehe ihn indiziert für linienförmige Knochenschnitte, Hemisektion, knöcherne axiale Perforation, zur crestalen Öffnung des Kieferkamms oder bei der Resektion von Wurzelspitzen. Ganz besonders wertvoll ist er für mich für die vorsichtige Zahnentfernung zur Vermeidung einer Osteotomiewunde geworden. Ziel muss es beim Lösen eines Zahns aus dem Alveolarfach sein, mit dem Fräser innerhalb des Zahns bzw. der Wurzel zu bleiben. Da stoße ich mit den Dimensionen eines herkömmlichen Lindemannfräsers oft an Grenzen. Mit dem neuen Frä-

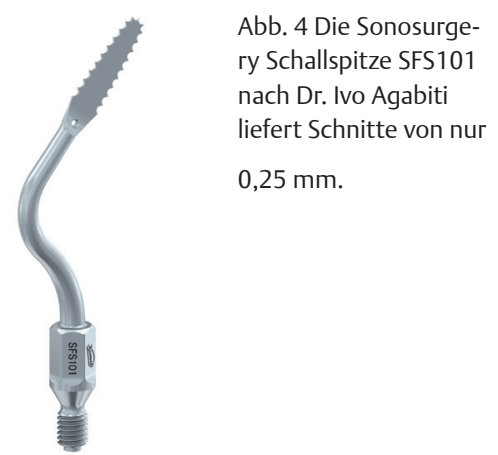

ser hingegen kann ich den Zahn bzw. die Wurzel sauber in 4 Quadranten zerlegen und die Zahnsplitter anschließend mit einem Handinstrument oder einer Pinzette 
vorsichtig herausholen. Auf diese Weise erhalte ich eine saubere Wunde, ohne dabei Knochensubstanz touchiert zu haben. Beste Voraussetzungen für eine Sofortimplantation!

Sind das nicht Indikationen, die auch per Schall z.B. mit den Schallspitzen nach Ivo Agabiti umsetzbar wären?

Blume: Ja, das wäre wohl der minimalinvasivste Weg, denn mit Knochenschnitten von nur 0,25 mm sind die SFS100-102 einfach unschlagbar. Die Schnitte sind hauchdünn, man kann extrem gezielt und kontrolliert vorgehen. Aber ich möchte nicht unerwähnt lassen, dass mit Schall der Mehraufwand höher ist in Bezug auf Zeit und Investition in einen eigenen Antrieb. Da komme ich mit rotierenden Instrumenten sehr viel effizienter und auch günstiger voran. Sagen wir es so: Die Schallspitzen nach Dr. Agabiti sind die aufwendige Königsklasse und insbesondere im Rahmen der Kammextensionstechniken ohne Auflagerungsplastik wie dem Bone Splitting/-spreading/-condensing meiner Ansicht nach unverzichtbar. Mir persönlich liefern die grazilen Formen des H255E und H254E sehr gute Voraussetzungen für ein substanzschonendes Arbeiten im Knochen auf rotierende Weise, wenn es um die herkömmlichen Indikationen wie Knochenschnitte oder Zahnteilung geht.

\section{Frage: Welchen Tipp geben Sie} den Kollegen, die den H255E zum 1. Mal in Händen halten?

Blume: Der Hersteller gibt ausreichend externe Kühlung mit mindestens $50 \mathrm{ml} /$ min vor, damit das Knochengewebe nicht zu stark erwärmt wird und Knochenspäne gut abgespült werden. Bei der Präparation achte ich auf geringen Druck (2N). Und ich denke, es versteht sich von selbst, dass das Instrument nicht als Hebel eingesetzt werden darf.

Vielen Dank für das Gespräch.

Das Interview führte Dorothee Holsten

Kontakt:

Zahnchirurgie im Franziskanerhof

Dres. Blume, Ziggel und Engelke

Uhlstraße 19-23

50321 Brühl

Tel: 02232-943486

www.zahnchirurgie-bruehl.de 